University of Wollongong

Research Online

Faculty of Social Sciences - Papers (Archive) Faculty of Arts, Social Sciences \& Humanities

2014

Infant face interest is associated with voice information and maternal

psychological health

Gemma Taylor

Lancaster University

Pauline Slade

University of Liverpool

Jane S. Herbert

University of Sheffield, herbertj@uow.edu.au

Follow this and additional works at: https://ro.uow.edu.au/sspapers

Part of the Education Commons, and the Social and Behavioral Sciences Commons

Research Online is the open access institutional repository for the University of Wollongong. For further information contact the UOW Library: research-pubs@uow.edu.au 


\title{
Infant face interest is associated with voice information and maternal psychological health
}

\author{
Abstract \\ Early infant interest in their mother's face is driven by an experience based face processing system, and is \\ associated with maternal psychological health, even within a non clinical community sample. The present \\ study examined the role of the voice in eliciting infants' interest in mother and stranger faces and in the \\ association between infant face interest and maternal psychological health.Infants aged 3.5-months were \\ shown photographs of their mother's and a stranger's face paired with an audio recording of their \\ mother's and a stranger's voice that was either matched (e.g., mother's face and voice) or mismatched \\ (e.g., mother's face and stranger's voice). Infants spent more time attending to the stranger's matched \\ face and voice than the mother's matched face and voice and the mismatched faces and voices. Thus, \\ infants demonstrated an earlier preference for a stranger's face when given voice information than when \\ the face is presented alone. In the present sample, maternal psychological health varied with $56.7 \%$ of \\ mothers reporting mild mood symptoms (depression, anxiety or stress response to childbirth). Infants of \\ mothers with significant mild maternal mood symptoms looked longer at the faces and voices compared \\ to infants of mothers who did not report mild maternal mood symptoms. In sum, infants' experience \\ based face processing system is sensitive to their mothers' maternal psychological health and the \\ multimodal nature of faces.

\section{Disciplines} \\ Education | Social and Behavioral Sciences
}

\section{Publication Details}

Taylor, G., Slade, P. \& Herbert, J. S. (2014). Infant face interest is associated with voice information and maternal psychological health. Infant Behavior and Development, 37 (4), 597-605. 
RUNNING HEAD: Infant Face Interest

Infant Face Interest is Associated with Voice Information and Maternal Psychological Health

Gemma Taylor $^{\mathrm{a}}$, Pauline Slade ${ }^{\mathrm{b}}$, and Jane S Herbert ${ }^{\mathrm{a}}$

a Department of Psychology, University of Sheffield, Sheffield, S10 2TN, UK

${ }^{\mathrm{b}}$ Clinical Psychology, Univeristy of Liverpool, Liverpool, L69 3GB, UK

$\underline{\text { Authors' Notes }}$

This research was supported by an ESRC studentship (ES/1018786/1) to the first author. The authors would like to thank all the infants and mothers who participated in this project. P. Slade is now at Liverpool University, UK. G. Taylor is now at Binghamton University, USA. Corresponding author: Dr. Jane S. Herbert, Department of Psychology, University of Sheffield, Sheffield S10 2TN, United Kingdom. Phone: +44 (0) 114222 6512, Fax: +44 (0) 114276 6515, email: j.s.herbert@ sheffield.ac.uk

\section{Highlights}


- Infant face interest is driven by experience

- We examined voices and maternal mood on infants' mother and stranger face interest

- 3.5-month olds show an earlier preference for stranger faces with voice information

- $56.7 \%$ of mothers reported mood symptoms associated with infant face/ voice interest

- Infants' face interest is sensitive to maternal mood and voice information

\section{Abstract}


Early infant interest in their mother's face is driven by an experience based face processing system, and is associated with maternal psychological health, even within a non clinical community sample. The present study examined the role of the voice in eliciting infants' interest in mother and stranger faces and in the association between infant face interest and maternal psychological health.

Infants aged 3.5-months were shown photographs of their mother's and a stranger's face paired with an audio recording of their mother's and a stranger's voice that was either matched (e.g., mother's face and voice) or mismatched (e.g., mother's face and stranger's voice). Infants spent more time attending to the stranger's matched face and voice than the mother's matched face and voice and the mismatched faces and voices. Thus, infants demonstrated an earlier preference for a stranger's face when given voice information than when the face is presented alone. In the present sample, maternal psychological health varied with $56.7 \%$ of mothers reporting mild mood symptoms (depression, anxiety or stress response to childbirth). Infants of mothers with significant mild maternal mood symptoms looked longer at the faces and voices compared to infants of mothers who did not report mild maternal mood symptoms. In sum, infants' experience based face processing system is sensitive to their mothers' maternal psychological health and the multimodal nature of faces. 


\section{Introduction}

Faces represent a special stimulus for preverbal infants, providing them with an early source of communication (see Nelson, 2001). Indeed, infants possess an innate face interest mechanism which facilitates early attention to faces (Morton \& Johnson, 1991) and drives an early preference for looking at their mother's face over a stranger's face (e.g., Bushnell, 2001; Field, Cohen, Garcia \& Greenberg, 1984; Pascalis, de Schonen, Morton, Deruelle, \& Fabre-Grenet, 1995). This preference, which is present within the first days of life, enables the formation of the mother-infant relationship which is important for infant's social and emotional development (Bowlby, 1969; Blass \& Camp, 2003). At around 2-months of age, Morton and Johnson (1991) propose that infant face interest is driven by an experience based system that is dependent upon infants' exposure to faces. Although early mother face interest has been widely researched, less is known about the experiences that may influence interest in mother and stranger faces as the infant develops.

In accordance with an experience based face processing system, infants prefer to look at faces that are the same gender (Quinn, Yahr, Kuhn, Slater \& Pascalis, 2002) and ethnicity (Kelly, Quinn, Slater, Lee, Gibson, Smith \& Pascalis, 2005) as their primary caregiver. Moreover, infants typically exhibit a developmental trend from a preference for looking towards their mother's face, when it is presented simultaneously with a stranger's face, to a preference for looking towards a stranger's face at around 4- to 5-months of age (Bartrip, Morton \& De Schonen, 2001). However, 3-month-old infants begin exhibiting a stranger face preference following repeated exposure to the photograph of their mother's face (Barerra \& Maurer, 1981). Thus, the trend from a mother face preference to a stranger face preference

around 4- to 5-months of age is assumed to reflect infants' increasing exposure and familiarity with their mother's face as well as an increasing drive toward attending to novel 
faces. Thus, individual differences in infant face interest may potentially reflect infants' prior experiences with their mother.

Infants' prior experiences with their mother may be shaped by their mother's maternal psychological health. Differences in infants' face interest have been documented in relation to maternal symptoms of postnatal depression (PND; for review, see Field, Diego \& Hernandez-Reif, 2009). Neonates of mothers with symptoms of depression take longer to habituate to their mother's face and voice (Hernandez-Reif, Field, Diego \& Largie, 2002) and, in general, orient less to faces and voices than neonates of mothers who do not report symptoms of depression (Hernandez-Reif, Field, Diego \& Ruddock 2006b). These findings persevere across early infancy; infants of mothers with symptoms of depression take longer to habituate to video clips of a stranger's happy/sad facial and vocal expressions (HernandezReif, Field, Diego, Vera \& Pickens, 2006a), spend less time attending to a stranger's live facial expressions (Diego, Field, Jones, Hernandez-Reif, Cullen, Schanberg et al., 2004) and fail to discriminate between a stranger's happy and neutral facial expressions (Bornstein, Atterberry, Mash \& Manian, 2011). Thus, there is an important influence of maternal symptoms of PND on infant face interest which persists across early infancy. The overarching aim of the present experiment was to determine the role of maternal psychological health as a prior social experience on infant's interest in their mother's and a stranger's face.

Maternal psychological health varies considerably within general community samples of postnatal women. Clinically significant levels of symptoms of PND are prevalent in around $17 \%$ of women (e.g., Morrell, Slade, Warner, Paley, Dixon, Walters et al., 2009) and symptoms of anxiety and post-traumatic stress disorder (PTSD) can also occur postnatally (Czarnocka \& Slade, 2000; Wenzel, Haugen, Jackson \& Brendle, 2005; White, Matthey, Boyd \& Barnett, 2006). For 3-5.6\% of mothers, childbirth is experienced as a traumatic event 
which results in clinical symptoms of PTSD (Creedy, Shochet \& Horsfall, 2000; Czarnocka \& Slade, 2000). Moreover, the mother-infant relationship is affected by maternal symptoms of PTSD (Davies, Slade, Wright \& Stewart, 2008; McDonald, Slade, Spiby \& Iles, 2011) and PND (Edhborg, Lundh, Seimyr \& Widstorm, 2001; Murray, 1992; Murray, Fiori-Cowley, Hooper \& Cooper, 1996; Taylor, Atkins, Kumar, Adams \& Glover, 2005). Thus, it is important to consider the role of a variety of maternal emotional responses, in relation to infant face interest, within general community samples.

Within such a community sample, Jones, Slade, Pascalis and Herbert (2013) have recently shown that differences in maternal psychological health affect infant face interest at 3.5-months of age. Maternal psychological health was determined in that study by mothers' responses to questionnaires regarding anxiety, depression, and stress responses to childbirth. An additional questionnaire also examined mother-infant bonding, which was determined by maternal perceptions of their infant's warmth and invasiveness. Infants were sequentially shown photographs of their mother's and a stranger's face, while the duration of visual attention to each face was measured. In this community sample, 57\% of mothers reported mild symptoms on at least one measure of anxiety, depression, or stress responses to childbirth. Overall, infants of mothers who did not report maternal mood symptoms looked longer during the presentation of their mother's face. In contrast, there was no difference in looking times to the mother's and a stranger's face by infants of mothers who report maternal mood symptoms. Moreover, maternal anxiety predicted infant interest in their mother's face such that increases in maternal symptoms of anxiety were associated with shorter infant looking times at their mother's face. Maternal perceptions of their infant's warmth and invasiveness, however, were not related to infant face interest. Taken together, the findings from Jones et al. (2013) emphasise how even small differences in maternal psychological 
health found within community samples can produce sufficiently different experiences to impact on infant face interest at 3.5 months of age.

Although Jones et al. (2013) have started to investigate how a variety of maternal factors in a community sample may interact with infant face interest, their research is not representative of infants' typical encounters with faces as it does not reflect the visual and auditory multidimensionality involved in face presentation in the normal environment (e.g., Brookes, Slater, Quinn, Lewkowicz, Hayes \& Brown, 2001; Coulon, Guella \& Streri, 2011; Sai, 2005). Consistent with the face interest literature, newborn infants exhibit a preference for listening to their mother's voice, rather than a female stranger's voice (DeCasper \& Fifer, 1980), and for listening to speech in their mother's language (Moon, Cooper \& Fifer, 1993). Moreover, early experience with the mother's voice from birth is necessary for facilitating neonatal mother face preference (Sai, 2005). Infants also orient to their mother's face when listening to a recording of her voice (Spelke \& Owsley, 1979). Thus, for young infants, experience with their mother's voice appears to be important for early mother face interest.

For younger infants, stranger face interest is determined by experience with voices. Infants' increased attention to dynamic talking faces over static silent faces may be explained by infants preference for dynamic stimuli over static stimuli (e.g., Shaddy \& Colombo, 2004; Ting \& Bergeson, 2007). Newborn infants recognise and show a preference for looking at a stranger's face that was previously seen talking over an unfamiliar talking face (Coulon et al., 2011). Given that all of the stimuli seen during familiarisation and test in Coulon et al.'s study were dynamic talking faces, a familiarity preference for a previously seen talking face may suggest that infants are trying to continue the interaction. Moreover, 3-month old infants look significantly longer at stranger's faces when they are presented in novel face/voice combinations (i.e., when the faces were presented with voices that weren't originally paired with that face) than familiar face/voice combinations (Brookes et al., 2001). At older ages, 
voices play less of a role in face interest, with visual novelty driving infants' attention. In Cohen (1974), 5- and 8-month old infants looked longest at a stranger's face, rather than their mother's face, regardless of whether each face was miming to the matched voice (e.g., stranger's voice and stranger's face) or mismatched voice (e.g., stranger's face and mother's voice). Greater interest in the stranger's face by 5-and 8-month old infants, even when the mother's voice was playing, suggests a strong preference for visual novelty at this age. Thus, voice information may contribute to individual differences in early infant face interest.

In the present experiment individual differences in early infant face interest at 3.5months of age were explored using mother and stranger faces that were matched and mismatched with the mother's and stranger's voice. The purpose of the mismatched faces was to determine the contributions of the mother's and the stranger's face and voice on infant face interest separately (see Cohen, 1974). Infants show a visual preference for their mother's face until around 5-months of age (Bartrip et al., 2001) and at 3-months of age also show a visual preference for novel stranger face and voice pairings (Brookes et al., 2001). It was hypothesised therefore, that although infants' would spend a long time attending to the stranger's matched face and voice and to the mismatched mother and stranger faces and voices, infants would look longest at their mother's face when it was presented with her voice.

The potential contribution of maternal factors to individual differences in infant face interest was explored based on maternal responses to questionnaires regarding: anxiety, depression, stress responses to childbirth, mother-infant attachment, and maternal well-being. It was hypothesised that mothers reporting more symptoms of depression, anxiety, and stress responses to childbirth would also report lower levels of mother-infant attachment and lower well-being. Moreover, infant face interest was hypothesised to be associated with maternal variables reflecting the experience based face processing system present during infancy 
(Morton \& Johnson, 1991). Consistent with Jones et al. (2013) it was hypothesised that infants of mothers who do not report experiencing symptoms of anxiety, depression and stress responses to childbirth would look longer at their mother's matched face. In contrast, consistent with Hernandez-Reif et al. (2006a) infants of mothers who report mild symptoms or above were hypothesised to show longer looking time to the stranger's matched face and voice. Given that the mismatched faces and voices have either the highly familiar mother's face or voice, attention to both of the mismatched faces and voices was expected to be influenced equally by maternal variables if both faces and voices drive attention equally (Bartrip et al., 2001).

\section{Method}

\section{Participants}

Participants were 60 mother-infant dyads selected from a community sample of mothers $(m=31.6$ years, $S . D=5.1$, range $=22-44)$ recruited from a local maternity ward following the birth of their infant. Infants were tested within 10 days of their 14-week birthdays (around 3.5-months; 33 females and 27 males). None of the infants were born prematurely ( $<37$ weeks gestation), required special care after birth, or had any identified hearing difficulties. The majority of mother-infant dyads were Caucasian (white British, $n=$ 57; white other, $n=1$, not reported $n=2$ ) and all mothers were English speaking with a regional accent to enable matching with the stranger's voice. The majority of mothers were educated to Degree level or higher (highest level of education; Degree or higher $n=34$, below graduate level $n=20$, not reported $n=6$ ).

An additional 15 mother-infant dyads were excluded from the sample due to experimenter error $(n=1)$, infant fussiness $(n=11)$ or infant drowsiness $(n=3)$. This $20 \%$ 
attrition rate is consistent with prior work using a similar procedure with this age group (e.g., $24.5 \%$ Bartrip et al., 2001; 8.6\% Brookes et al., 2001; 28.9\% Jones et al., 2013).

\section{Face/Voice Stimuli}

For each infant, the stimuli consisted of a photograph of their mother's face, along with an audio recording of her voice, and a photograph of a female stranger's face, along with an audio recording of her voice. The photograph of the mother's face was taken upon arrival for the study and roughly matched for age, hair colour and the presence of glasses to a photograph of a stranger's face selected from a database of female faces. In all photographs, the woman held a neutral facial expression and her clothing was covered with a white lab coat. The photograph was taken against a plain cream background and then cropped to exclude extraneous shoulder detail and re-sized on Microsoft PowerPoint. The face stimuli were $17.5 \mathrm{~cm}(\mathrm{w}) \times 18.5 \mathrm{~cm}(\mathrm{~h})$ when shown on the 17 inch flat panel monitor used during the test session.

Each mother's voice was audio recorded in an anechoic chamber on the day of their visit. An Olympus WS-450S digital voice recorder was used to record the mother as she read a short rhyming story book to her infant ("When I go to the Park", Jill Harker, 2002). If the infant was clearly audible on the recording it was re-recorded. The stranger's voice was recorded under the same conditions. The same stranger's voice recording was presented to all infants in order to control for differences in the length of the recording and in voice quality, pitch and intonation (see Bettes, 1988; Scherer, 1986).

The voice recordings were uploaded onto the test computer and added to the PowerPoint presentation so that the face/voice presentation occurred in synchrony. The face/voice stimuli were entered sequentially onto a PowerPoint presentation so that the faces and voices were first presented in a matched protocol (mother's face and voice together, 
stranger's face and voice together) followed by the mismatched protocol (mother's face and stranger's voice, stranger's face and mother's voice). A grey screen presented for 2 seconds separated the presentation of each face/voice combination (see Figure 1). The order of presentation of the mother and stranger's face was counterbalanced across infants within the matched and mismatched phases.

\section{Measures}

Hospital Anxiety and Depression Scale (HADS; Zigmond \& Snaith, 1983): This 14 item questionnaire measures symptoms of depression and anxiety experienced over the past 7 days. Cronbach's alpha for the 7 anxiety and 7 depression items were 0.82 and 0.83 respectively (Bjelland, Dahl, Haug \& Neckelmann, 2002). The subscales were scored using the following cut-off points in line with clinical usage: scores of 0-7 are considered examples of no clinical symptoms, 8-10 for mild levels of clinical symptoms, 11-15 for moderate levels of clinical symptoms, and 16+ for severe levels of clinical symptoms.

Impact of Events Scale (IES; Horowitz, Wilner \& Alvarez, 1979): For the purpose of the present study, the specified stressor identified on the IES was childbirth. The IES consists of two dimensions experienced in the past 7 days, a 7 item intrusion subscale (internal reliability $\alpha=0.85)$ and an 8 item avoidance subscale $(\alpha=0.85)$. Studies typically consider scores of 0 8 as 'sub-clinical', 9-25 'mild reaction to trauma', 26-43 'moderate', and 44+ 'severe' (e.g., Chisholm, Freeman \& Cooke, 2006).

Maternal Postnatal Attachment Scale (MPAS; Condon \& Corkindale, 1998): This 19 item self-report questionnaire provides a measure of mother-infant attachment $(\alpha=0.78)$. The questions assessed quality of attachment (e.g., "Regarding my level of interaction with the baby I: feel very / moderately / slightly guilty that I am not more involved / I don't have any guilty feelings regarding this"), absence of hostility (e.g., "Regarding the things that we have 
had to give up because of the baby: I find that I resent it quite a lot / a moderate amount / a bit / I don't resent it at all") and pleasure in interaction ("When I am with the baby: I usually try to prolong the time I spend with him/her / I usually try to shorten the time I spend with him/her). Overall, higher scores (19-95) suggest higher attachment ratings. Internal reliability from Cronbach's alpha was .86 for the present sample.

Warwick-Edinburgh Mental Well-being Scale (WEMWBS; Tennant, Hiller, Fishwick, Platt, Joseph, Weich et al., 2007): This 14 item questionnaire was chosen to measure mother's mental well-being. The questionnaire consists of positively worded statements such as, "I've been feeling good about myself" and "I've had energy to spare" scored on a 5 point scale from none of the time to all of the time. While Tennant et al., do not describe a cut-off point, high scores (range: 14-70) indicate a greater level of mental well-being. For the present sample, reliability from Cronbach's alpha was .90 .

\section{Procedure}

Infants were tested in the developmental lab at a time of day the mother identified as an alert/awake period. Upon arrival, the purpose of the study was explained to the mother and informed consent was obtained. A photograph of the mother's face was taken and she was then ask to complete the four questionnaires (HADS, IES, MPAS and WEMWBS) while the experimenter processed the photograph to create the mother face stimuli and matched it to a stranger's face. The questionnaires were marked with a participant number and sealed in an envelope so that they remained anonymous to the experimenter. The questionnaires remained anonymous until after all infant data had been coded. The mother's voice was recorded in the experimental room and immediately uploaded onto the PowerPoint presentation. The recording took around 3 minutes to upload. During this time, the mother was provided with toys for her infant and they were free to move around and interact together. 
For the test session, the mother sat with the infant on her lap facing a computer screen approximately $60 \mathrm{~cm}$ away. The room was darkened and mothers were instructed not to speak to their infant during the session or to direct his/her attention in any way. The presentation started with a welcome screen to attract infant's attention. All infants were then presented with the two matched images (mother's face and voice, stranger's face and voice), followed by the two mismatched images (mother's face and stranger's voice, stranger's face and mother's voice; see Figure 1). Each face was shown individually for the duration of the voice recording, approximately 60 seconds. The order of presentation (mother face or stranger face first) for each set of images was counterbalanced across infants. The entire procedure lasted around 5 minutes.

A video camera situated above the presentation screen was used to record infant looking behaviour to each face during the session for later analysis. Videos were coded frame by frame (25 per second) in VP Video Lab to determine looking time to each face and voice presentation. All videos were scored double blind and approximately $25 \%(n=16)$ of the videos were double coded by a second independent experimenter. Infant looking times, as coded by the two experimenters, were highly correlated, $\operatorname{ICC}(2,2)$, absolute $=.98$ (see Shrout \& Fleiss, 1979). The maternal questionnaires were also double entered by the second experimenter to ensure accuracy. Any disagreements were resolved through discussion.

\section{Results}

The aims for the following analyses were threefold: 1) to examine infant attention to their mother's face and voice, a stranger's face and voice and mismatched mother and stranger faces and voices, 2) to explore the relationship between maternal mood (anxiety, depression, stress reaction to childbirth), perceived mother-infant attachment and maternal well-being, and 3), to determine whether maternal mood predicts infant face and voice 
interest. Maternal variables were assessed both as a dichotomy and as a continuum to allow for analysis across the spectrum.

\section{Infant Face Interest}

Three infants failed to look at the mismatched stranger's face and mother's voice for more than 10 seconds and so were excluded from analyses for the mismatched stranger's face and mother's voice only.

Preliminary analyses found no significant effect of presentation order or infant gender on infant face interest. The length of voice recordings by the mothers $(M=51.45 \mathrm{~s}, S . D=$ 8.61 , range $=39 \mathrm{~s}-89 \mathrm{~s})$ was significantly shorter than the stranger's voice recording $(57 \mathrm{~s} ; t$ $(59)=-5.00, p=.000)$, however, the length of the mothers' voice recordings were not correlated to infant face interest. As such, the data for infant face interest was collapsed across gender, presentation order and the length of the voice recording.

To determine whether infant looking times differed according to the mother and stranger face and voice combinations, a one-way repeated measures ANOVA was conducted. Overall, there was a significant effect of face and voice combination on infant face interest, $F$ $(3,168)=9.31, p=.000$. Infants attended to the stranger's matched face and voice $(M=$ 50.41 s, $S . D=8.84$ ) for longer than the other three conditions: mother's matched face and voice $(M=42.15$ s, $S . D=11.07, p=.000)$, mismatched mother's face and stranger's voice $(M=44.54 \mathrm{~s}, S . D=11.77, p=.004)$ and mismatched stranger's face and mother's voice $(M$ $=43.10 \mathrm{~s}, S . D=11.00, p=.000$ ). All other comparisons were non-significant (all $p$ values $=$ 1.00). Thus, 3.5-month old infants looked longer at the stranger's matched face and voice rather than their mother's matched face and voice.

\section{Maternal Variables}


Complete maternal questionnaires were available for 57 mothers. The remaining 3 mothers omitted more than $20 \%$ of the questions on one of the four measures (WEMWBS $n$ $=1$, MPAS $n=2$ ) due to overlooking whole a page of the questionnaire and so do not contribute to data on these individual measures.

The questionnaire data were initially screened for skewness and kurtosis. The HADS depression scale had a significant positive skew and was transformed using a log transformation. The IES had a significant positive skew and kurtosis. Therefore this variable was transformed into a dichotomous variable (mild symptoms, scores of 9 or above, versus no symptoms) to account for the large number of participants scoring zero, which are to be expected in a non-clinical population. Finally, the MPAS had a significant negative skew and was reverse scored and transformed using a log transformation. As such, high scores on the transformed T-MPAS now represent lower perceived attachment whilst low scores on the TMPAS represent higher perceived attachment. Descriptive analyses for the maternal questionnaire data are shown in Table 1. To determine the relationship between the maternal variables, correlation analyses were conducted. Overall, the HADS, WEMWBS and T-MPAS scores were correlated with one another, such that low levels of maternal anxiety were correlated with low levels of maternal depression and high levels of maternal well-being and greater perceived mother-infant attachment (see Table 2).

\section{Infant Face/Voice Interest and Maternal Variables}

To determine whether maternal mood predicts infant face and voice interest, four multiple regression analyses were conducted using a backward-stepwise method to establish whether maternal mood (HADS: anxiety, depression), maternal well-being (WEMWBS) or perceived mother-infant attachment (T-MPAS) predict infant interest in the matched and mismatched face and voice combinations. A backward-stepwise method was selected because 
the regression models were exploratory in nature, and also to reduce the likelihood of type 2 error. Infant interest in the stranger's matched face and voice was significantly predicted by the model incorporating perceived mother-infant attachment, $F=9.50, R^{2}=.147, p=.003$. Low perceived mother-infant attachment was associated with increased looking at the stranger's matched face and voice; however causality cannot be inferred from this analysis. For example, low perceived mother-infant attachment could be a consequence of infants' interest in stranger's faces or the cause of infants' interest in stranger's faces. In addition, infant interest in the mismatched mother's face and stranger's voice was significantly predicted by the model incorporating maternal anxiety, $F=7.45, R^{2}=.119, p=.008$. Higher maternal anxiety was associated with increased infant looking at the mother's face when a stranger's voice was playing. Infant interest in their mother's matched face and voice, and mismatched mother's face and stranger's voice were not significantly predicted by any of the maternal variables.

Using the criteria for normal, mild, moderate, and severe symptoms, the presence of maternal symptoms of anxiety, depression, and stress responses to childbirth (IES) was assessed as a dichotomy. Although the mothers were from a community sample, $56.7 \%$ of mothers $(n=34)$ reported having mild symptoms or above on at least one measure. The number of mothers reporting mild symptoms or above was highest for stress responses to childbirth $(n=27,45 \%)$, followed by anxiety $(n=24,40 \%)$ and depression $(n=5,8.3 \%)$. Moreover, a series of $t$-tests showed that mothers who reported symptoms of anxiety, depression, or stress responses to childbirth also reported lower levels of maternal well-being $(M=48.80, S . D=7.56, t(57)=-3.87, p=.000)$ and perceived mother-infant attachment (MPAS $M=80.57, S . D=8.61, t(56)=-2.95, p=.005)$ compared to mothers who did not report symptoms (maternal well-being, $M=56.16, S . D=6.73$; perceived mother-infant attachment, MPAS $M=86.58, S . D=6.26$ ). 
To determine whether face/voice interest also differed as a function of the mother having mild symptoms or above on at least one measure of anxiety, depression or stress responses to childbirth, a two-way repeated measures ANOVA was conducted. Overall, there was a significant main effect of symptom group, $F(1,55)=7.83, p=.007$. Infants of mothers reporting mild symptoms or above for either anxiety, depression, or stress responses to childbirth looked at the matched and mismatched faces and voices for significantly longer $(M$ $=47.28, S . D=5.94)$ than infants of mothers who did not report any symptoms $(M=42.20$, $S . D=7.77)$. There was no significant interaction effect between face/voice combination and maternal symptom group, $F(1,55)=0.21, p=.886$. Thus, maternal symptoms of anxiety, depression or stress response to childbirth are related to higher infant face and voice interest.

\section{Discussion}

Infants typically show a preference for their mother's face until 4- to 5-months of age when presented with faces only (e.g., Bartrip et al., 2001). However, in the present study, 3.5months old infants looked significantly longer at the matched stranger's face and voice compared to the matched mother's face and voice and mismatched mother and stranger faces and voices. In other words, infant attention to the stimuli decreased when the stimuli included either their mother's face or voice. Thus, the transition from a mother face preference to a stranger face preference in the present experiment may be driven, at least in part, by the additional voice information. Similarly, one potential driver for the earlier interest in the stranger's face when it is paired with a stranger's voice might be to enable infants to facilitate an interaction with a novel person (for similar argument, see Coulon et al., 2011). Given that infants in our study were sitting on their mother's lap during the test session, infants might

not have been driven to facilitate such an interaction with their mother by looking at the photograph of her face. This finding, therefore, supports the literature which suggests that 
voices play a role in infant face interest (e.g., Brookes et al., 2001; Coulon et al., 2011; Sai, 2005).

Infants' attention to the mismatched mother and stranger faces and voices was lower than the stranger's matched face and voice. This finding contrasts with Brookes et al. (2001) in which infants showed greater interest in novel stranger face and voice pairings. However in that study, infants were familiarised to novel stranger face and voice pairings before presenting them in novel combinations. Thus, both components of the mismatched stimuli were equally familiar to the infant. In contrast, in the present experiment, the mismatched face and voice stimuli contained one highly familiar component (e.g., the mother's face or voice) and one less familiar component (e.g., stranger's face or voice). Infants' reduced attention to the mismatched mother and stranger faces and voices in the present study may be due to infant's familiarity with their mother, consistent with an experience based face processing system (Morton \& Johnson, 1991). Moreover, prior research suggests that between 2- and 4-months of age, infants begin to use multi-modal information for face discrimination (Bahrick, Hernandez-reif \& Flom, 2005). Our findings of a strong novelty preference at 3.5-months of age are consistent with research by Uttley, Hillairet de Boisferon, Dupierrix, Lee, Quinn et al. (2013) in which infants showed a preference for looking at a novel other-race face and non-native language compared to a mismatched other-race face and the infants' native language. Thus, infants' experience with their mother may influence the strength of this novelty preference for a stranger's face and voice.

Infants of mothers who reported mild or above maternal mood symptoms looked longer at the faces than infants of mothers who did not report maternal mood symptoms. The finding that maternal psychological health interacts with infant face interest is consistent with the findings of Hernandez-Reif et al. (2006a), in which infants of mothers with symptoms of PND took longer to habituate to a video clip of a stranger's facial and vocal expressions. 
However, when faces are presented without additional voice or motion information, infants of mothers with maternal symptoms of anxiety, depression or stress responses to childbirth show equal attention to photographs of their mother's and a stranger's face (Jones et al., 2013) and decreased attention to a live stranger's face when mothers reported symptoms of PND (Diego et al., 2004). The drive to engage in rewarding social interactions may explain the association between maternal psychological health and infant face interest. Social interactions between infants and their mothers may be impaired if mothers report maternal mood symptoms (e.g., Beebe, Badalamenti, Jaffe, Feldstein, Marquette, Helbraun et al., 2008; Edhborg et al., 2001; Field, Healy, Goldstein, Perry, Bendell, Schanberg et al., 1988; Murray et al., 1996) and this may, therefore, drive infants to seek rewarding social interactions from others. Thus, we speculate infants of mothers with maternal mood symptoms might increase attention to faces paired with voices in order to facilitate a social interaction with their mother and stranger, the drive for which is not present when faces are presented alone.

In the present experiment, maternal psychological health predicted infant face interest when the stranger's voice was present. Specifically, perceived mother-infant attachment significantly predicted $14.7 \%$ of the variance in infant interest in the stranger's matched face and voice whilst maternal anxiety predicted $11.9 \%$ of the variance in infant interest in the mismatched mother's face and stranger's voice. The addition of voices might explain the disparity between the findings in the present experiment and those of Jones et al. (2013) who found that maternal psychological health predicted infant interest in their mother's face only. Research has found that voice quality, pitch and intonation are affected by maternal mood symptoms (Bettes, 1988; Scherer, 1986). Given that within community samples, mothers show significant variation in maternal psychological health (also see Jones et al., 2013), differences in voice quality may also occur. As a result, the association between maternal 
psychological health and infant face interest during the stranger's voice recording found in the present experiment might reflect the stranger's voice recording being more or less novel to the infant in terms of voice quality, pitch and/or intonation. In the present experiment, voice novelty appears to drive attention whilst in the Cohen (1974) study visual novelty appeared to drive attention at 5- and 8-months. In other words, the drivers of face interest may change across age, such that both the multi-modal nature of faces and infants previous experiences with their mother should be considered when assessing infant face interest.

Maternal psychological health varied considerably across our community sample. Around $56.7 \%$ of mothers in the present sample reported experiencing mild mood symptoms. Specifically, mothers primarily reported symptoms of a stress response to childbirth, followed by anxiety and depression. Although maternal psychological health in the present study was within a normal range for a community sample (see, Condon \& Corkindale, 1998; Crawford, Henry, Crombie \& Taylor, 2001; Davies et al., 2008; Jones et al., 2013; Tennant et al., 2007), maternal mood was negatively correlated with perceived mother-infant attachment (see also Davies et al., 2008). This correlation suggests that perceived mother-infant attachment might be a more appropriate measure of the mother-infant relationship than maternal perceptions of their infant's warmth and invasiveness used by Jones et al., (2013). The relationship between maternal mood and the mother-infant relationship has been well documented in samples of mothers with PND (e.g., Murray et al., 1996; Taylor et al., 2005). However, the current findings suggest that even small differences in maternal mood are associated in differences of perceived mother-infant attachment.

The high prevalence of maternal mood symptoms in community samples warrants further research to determine the extent to which the mother-infant relationship is affected and the subsequent impact this may have on infant development. Indeed, given that maternal psychological health as measured by maternal mood measures predicted $11-15 \%$ of the 
variance in infant face interest in the present study, future work should consider the extent to which the remaining variance in infant face interest may be explained by the behavioural impact of maternal mood. For example, a freeplay session may capture a more naturalistic representation of the role of maternal mood on the mother-infant relationship and may subsequently be a stronger predictor of infant face interest. Given that early infant interest in their mother's face is highly important for infant's social and emotional development (Bowlby, 1969; Blass \& Camp, 2003), this question represents a important area for future research.

In conclusion, infants' experience based face processing system is particularly sensitive to the infant's immediate environment such that small differences in maternal psychological health can contribute to individual differences in infant face interest. Moreover, multimodal features such as voice information interact with maternal psychological health and infant face interest across age. One potential reason for the association between maternal psychological health and infant face interest may be to enable infants to facilitate rewarding social interactions which may be impaired between infants and their mothers if mothers report maternal mood symptoms (e.g., Beebe et al., 2001; Edhborg et al., 2001; Field et al., 1988; Murray et al., 1996). Understanding how attention to faces changes as a function of age and experience early on in development may also be important for investigating the link between maternal psychological health and infant cognitive development (e.g., Beck, 1998). 


\section{References}

Bahrick, L. E., Hernandez-Reif, M., \& Flom, R. (2005). The development of infant learning about specific face-voice relations.Developmental Psychology, 41, 541-552.

Barrera, M., \& Maurer, D. (1981). Recognition of mother's photographed face by the three-month-old infant. Child Development, 52(2), 714-716.

Bartrip, J., Morton, J., \& De Schonen, S. (2001). Responses to mother's face in 3-week to 5-month-old infants. British Journal of Developmental Psychology, 19(2), 219-232.

Beck, C. T. (1998). The effects of postpartum depression on child development: a metaanalysis. Archives of Psychiatric Nursing, 12(1), 12-20.

Beebe, B., Badalamenti, A., Jaffe, J., Feldstein, S., Marquette, L., Helbraun, E., et al. (2008). Distressed mothers and their infants use a less efficient timing mechanism in creating expectancies of each other's looking patterns. Journal of Psycholinguistic Research, 37(5), 293-307.

Bettes, B. (1988). Maternal depression and motherese: Temporal and intonational features. Child Development 59(4), 1089-1096.

Bjelland, I., Dahl, A., Haug, T., \& Neckelmann, D. (2002). The validity of the Hospital Anxiety and Depression Scale: An updated literature review. Journal of Psychosomatic Research, 52(2), 69-77.

Blass, E., \& Camp, C. (2003). Biological bases of face preference in 6-week-old infants. Developmental Science, 6(5), 524-536. 
Bornstein, M. H., Arterberry, M. E., Mash, C., \& Manian, N. (2011). Discrimination of facial expression by 5-month-old infants of nondepressed and clinically depressed mothers. Infant Behavior and Development, 34(1), 100-106.

Bowlby, J. (1969). Attachment and Loss (Vol. 1). London: Hogarth Press.

Brookes, H., Slater, A., Quinn, P. C., Lewkowicz, D. J., Hayes, R., \& Brown, E. (2001). Three month old infants learn arbitrary auditory-visual pairings between voices and faces. Infant and Child Development, 10(1), 75-82.

Bushnell, I. W. R. (2001). Mother's face recognition in newborn infants: Learning and memory. Infant and Child Development, 10(1), 67-74.

Carpenter, M., Tomasello, M., \& Savage-Rumbaugh, S. (1995). Joint attention and imitative learning in children, chimpanzees, and enculturated chimpanzees. Social Development, 4(3), 217-237.

Chisholm, B., Freeman, D., \& Cooke, A. (2006). Identifying potential predictors of traumatic reactions to psychotic episodes. British Journal of Clinical Psychology, 45(4), 545559.

Cohen, S. (1974). Developmental differences in infants' attentional responses to facevoice incongruity of mother and stranger. Child Development, 45(4), 1155-1158.

Condon, J. T., \& Corkindale, C. J. (1998). The assessment of parent-to-infant attachment: development of a self-report questionnaire instrument. Journal of Reproductive and Infant Psychology, 16(1), 57-76.

Coulon, M., Guellai, B., \& Streri, A. (2011). Recognition of unfamiliar talking faces at birth. International Journal of Behavioral Development, 35(3), 282-287. 
Crawford, J., Henry, J., Crombie, C., \& Taylor, E. (2001). Normative data for the HADS from a large non-clinical sample. British Journal of Clinical Psychology, 40(4), 429434.

Creedy, D. K., Shochet, I. M., \& Horsfall, J. (2000). Childbirth and the development of acute trauma symptoms: incidence and contributing factors. Birth, 27(2), 104-111.

Czarnocka, J., \& Slade, P. (2000). Prevalence and predictors of post-traumatic stress symptoms following childbirth. British Journal of Clinical Psychology, 39(1), 35-51.

Davies, J., Slade, P., Wright, I., \& Stewart, P. (2008). Posttraumatic stress symptoms following childbirth and mothers' perceptions of their infants. Infant Mental Health Journal, 29(6), 537-554.

DeCasper, A., \& Fifer, W. (1980). Of Human Bonding: Newborns Prefer Their Mothers' Voices. Science, 208(4448), 1174-1176.

Diego, M. A., Field, T., Jones, N. A., Hernandez-Reif, M., Cullen, C., Schanberg, S., et al. (2004). EEG responses to mock facial expressions by infants of depressed mothers. Infant Behavior and Development, 27(2), 150-162.

Edhborg, M., Lundh, W., Seimyr, L., \& Widstrom, A. M. (2001). The long-term impact of postnatal depressed mood on mother-child interaction: a preliminary study. Journal of Reproductive and Infant Psychology, 19(1), 61-71.

Field, T. M., Cohen, D., Garcia, R., \& Greenberg, R. (1984). Mother-stranger face discrimination by the newborn. Infant Behavior and Development, 7(1), 19-25.

Field, T., Diego, M., \& Hernandez-Reif, M. (2009). Depressed mothers' infants are less responsive to faces and voices. Infant Behavior and Development, 32(3), 239-244. 
Field, T., Healy, B., Goldstein, S., Perry, S., Bendell, D., Schanberg, S., et al. (1988). Infants of depressed mothers show "Depressed" behavior even with nondepressed adults. Child Development, 59(6), 1569-1579.

Harker, J. (2000). When I go to the park. UK: Parragon Press.

Hernandez-Reif, M., Field, T., Diego, M., \& Largie, S. (2002). Depressed mothers' newborns show longer habituation and fail to show face/voice preference. Infant Mental Health Journal, 23(6), 643-653.

Hernandez-Reif, M., Field, T., Diego, M., \& Ruddock, M. (2006b). Greater arousal and less attentiveness to face/voice stimuli by neonates of depressed mothers on the Brazelton Neonatal Behavioral Assessment Scale. Infant Behavior and Development, 29(4), 594-598.

Hernandez-Reif, M., Field, T., Diego, M., Vera, Y., \& Pickens, J. (2006a). Happy faces are habituated more slowly by infants of depressed mothers. Infant Behavior and Development, 29(1), 131-135.

Horowitz, M., Wilner, N., \& Alvarez, W. (1979). Impact of Event Scale: A measure of subjective stress. Psychosomatic Medicine, 41(3), 209-218.

Jones, R., Slade, P., Pascalis, O., \& Herbert, J.S. (2013). Infant interest in their mother's face is associated with maternal psychological health. Infant Behavior and Development, 36(4), 686-693.

Kelly, D. J., Quinn, P. C., Slater, A. M., Lee, K., Gibson, A., Smith, M., et al. (2005). Three-month-olds, but not newborns, prefer own-race faces. Developmental Science, 8(6), F31-F36. 
McDonald, S., Slade, P., Spiby, H., \& Iles, J. (2011). Post-traumatic stress symptoms, parenting stress and mother-child relationships following childbirth and at 2 years postpartum. Journal of Psychosomatic Obstetrics and Gynecology, 32(3), 141-146.

Moon, C., Cooper, R. P., \& Fifer, W. P. (1993). Two-day-olds prefer their native language. Infant Behavior and Development, 16(4), 495-500.

Morrell, C. J., Slade, P., Warner, R. Paley, G., Dixon, S., Walters, S. J., Brugha, T., Barkham, M., Parry, G. J., \& Nicholl, J. (2009). Clinical effectiveness of health visitor training in psychologically informed approaches for depression in postnatal women: pragmatic cluster randomised trial in primary care. BMJ: British Medical Journal, 338: a3045.

Morton, J., \& Johnson, M. H. (1991). CONSPEC and CONLERN: a two-process theory of infant face recognition. Psychological Review; Psychological Review, 98(2), 164-.

Murray, L. (1992). The impact of postnatal depression on infant development. Journal of Child Psychology and Psychiatry, 33(3), 543-561.

Murray, L., Fiori-Cowley, A., Hooper, R., \& Cooper, P. (1996). The impact of postnatal depression and associated adversity on early mother-infant interactions and later infant outcome. Child Development, 67(5), 2512-2526.

Nelson, C. A. (2001). The development and neural bases of face recognition. Infant and Child Development, 10(1-2), 3-18.

Pascalis, O., de Schonen, S., Morton, J., Deruelle, C., \& Fabre-Grenet, M. (1995). Mother's face recognition by neonates: A replication and an extension. Infant Behavior and Development, 18(1), 79-85. 
Quinn, P., Yahr, J., Kuhn, A., Slater, A., \& Pascalis, O. (2002). Representation of the gender of human faces by infants: A preference for female. Perception, 31(9), 1109-1122.

Sai, F. Z. (2005). The role of the mother's voice in developing mother's face preference: Evidence for intermodal perception at birth. Infant and Child Development, 14(1), 29-50.

Scherer, K. (1986). Vocal affect expression: A review and a model for future research. Psychological Bulletin, 99(2), 143-165.

Shaddy, D. J., \& Colombo, J. (2004). Developmental changes in infant attention to dynamic and static stimuli. Infancy, 5(3), 355-365.

Spelke, E., \& Owsley, C. (1979). Intermodal exploration and knowledge in infancy. Infant Behavior and Development, 2, 13-27.

Strid, K., Tjus, T., Smith, L., Meltzoff, A., \& Heimann, M. (2006). Infant recall memory and communication predicts later cognitive development. Infant Behavior and Development, 29(4), 545-553.

Taylor, A., Atkins, R., Kumar, R., Adams, D., \& Glover, V. (2005). A new Mother-toInfant Bonding Scale: links with early maternal mood. Archives of Women's Mental Health, $8(1), 45-51$.

Tennant, R., Hiller, L., Fishwick, R., Platt, S., Joseph, S., Weich, S., et al. (2007). The Warwick-Edinburgh mental well-being scale (WEMWBS): development and UK validation. Health and Quality of Life Outcomes, 5(1), 63.

Ting, J.Y., Bergeson, T.R. (2007). Infant preference for dynamic versus static faces. Society for Research in Child Development Biennial Meeting, Boston, MA. 
Wenzel, A., Haugen, E. N., Jackson, L. C., \& Brendle, J. R. (2005). Anxiety symptoms and disorders at eight weeks postpartum. Journal of Anxiety Disorders, 19(3), 295-311.

Uttley, L., De Boisferon, A. H., Dupierrix, E., Lee, K., Quinn, P. C., Slater, A. M., \& Pascalis, O. (2013). Six-month-old infants match other-race faces with a non-native language. International Journal of Behavioral Development, 37(2), 84-89.

White, T., Matthey, S., Boyd, K., \& Barnett, B. (2006). Postnatal depression and posttraumatic stress after childbirth: Prevalence, course and co-occurrence. Journal of Reproductive and Infant Psychology, 24(02), 107-120.

Zigmond, A. S., \& Snaith, R. P. (1983). The Hospital Anxiety and Depression Scale. Acta Psychiatrica Scandinavica, 67(6), 361-370. 
Figure 1.

An example of the face/voice presentation and the duration of presentation for each slide.

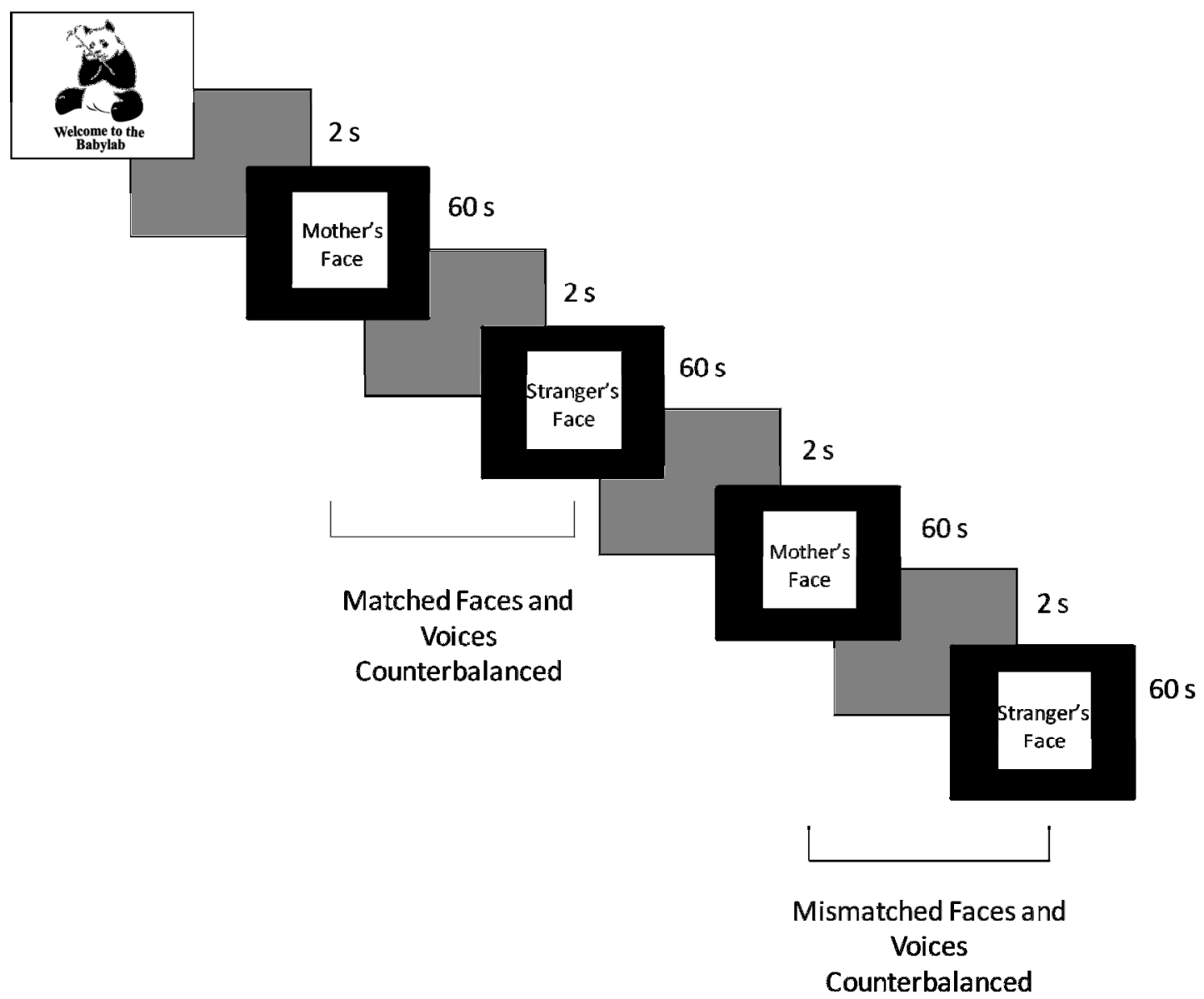


Table 1.

Descriptive statistics for maternal questionnaires

\begin{tabular}{lcccc}
\hline Variable & Sample & Mean & Standard & Potential \\
& Size & & Deviation & Range \\
& & & & \\
\hline Anxiety (HADS) & 60 & 6.57 & 3.12 & $0-21$ \\
Depression (HADS) & 60 & 3.73 & 2.28 & $0-21$ \\
Stress reaction to childbirth (IES) & 60 & 9.15 & 11.30 & $0-75$ \\
$\quad$ Intrusion & & 5.50 & 6.54 & $0-35$ \\
$\quad$ Avoidance & 58 & 83.16 & 8.19 & $19-95$ \\
Maternal postnatal attachment (MPAS) & & 41.30 & 3.26 & $9-45$ \\
$\quad$ Quality of attachment & & 19.62 & 3.64 & $5-25$ \\
$\quad$ Absence of hostility & & 22.24 & 2.57 & $5-25$ \\
$\quad$ Pleasure in interaction & & 51.92 & 8.05 & $14-70$ \\
Well-being (WEMWBS) & 59 & & & \\
\hline
\end{tabular}


Table 2.

Correlations between maternal variables; anxiety, depression, well-being, maternal attachment

\begin{tabular}{lccc}
\hline & Anxiety (HADS) & Depression (HADS) & T-MPAS \\
\hline Depression (HADS) & $.466^{*}$ & & \\
T-MPAS & $.487^{*}$ & $.467^{*}$ & \\
WEMWBS & $-.564^{*}$ & $-.530^{*}$ & $-.676^{*}$ \\
\hline
\end{tabular}

Note. $* p<.001$ 